Supporting Information for

\title{
Selectivity Control in Tandem Catalytic Furfural Upgrading on Zeolite Encapsulated Pt Nanoparticles through Site and Solvent Engineering
}

\author{
Hong Je Cho, Doyoung Kim, and Bingjun $\mathrm{Xu}^{*}$ \\ Department of Chemical and Biomolecular Engineering, University of Delaware, Newark, \\ Delaware 19716, United States \\ *E-mail: $\underline{\text { bxu@udel.edu }}$
}



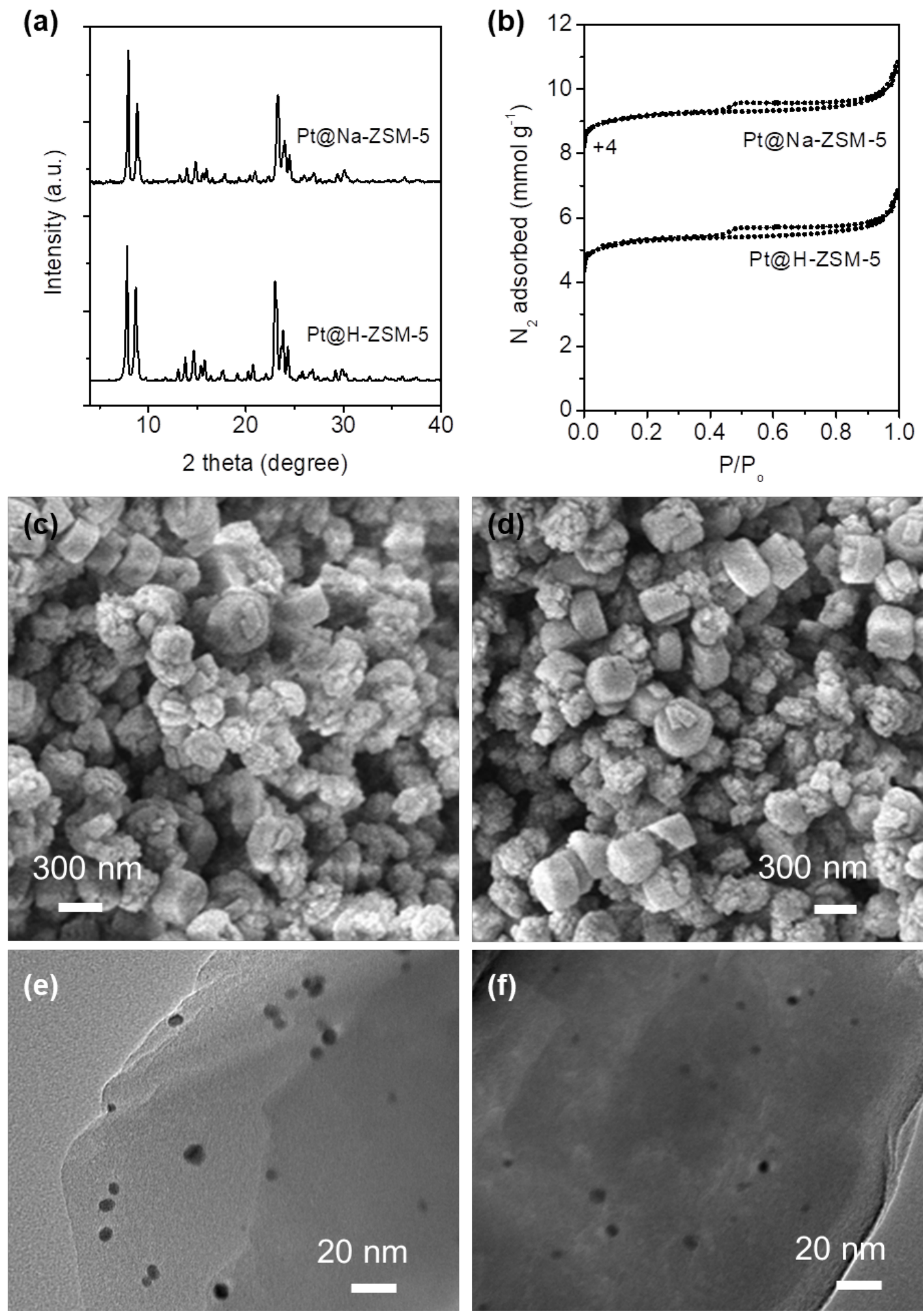

Figure S1. (a) XRD patterns and (b) $\mathrm{N}_{2}$ physisorption isotherms of Pt@Na-ZSM-5 and Pt@H-ZSM-5 samples, SEM images of (c) Pt@Na-ZSM-5 and (d) Pt@H-ZSM-5, and TEM images of (e) Pt@Na-ZSM-5 and (f) Pt@H-ZSM-5. 

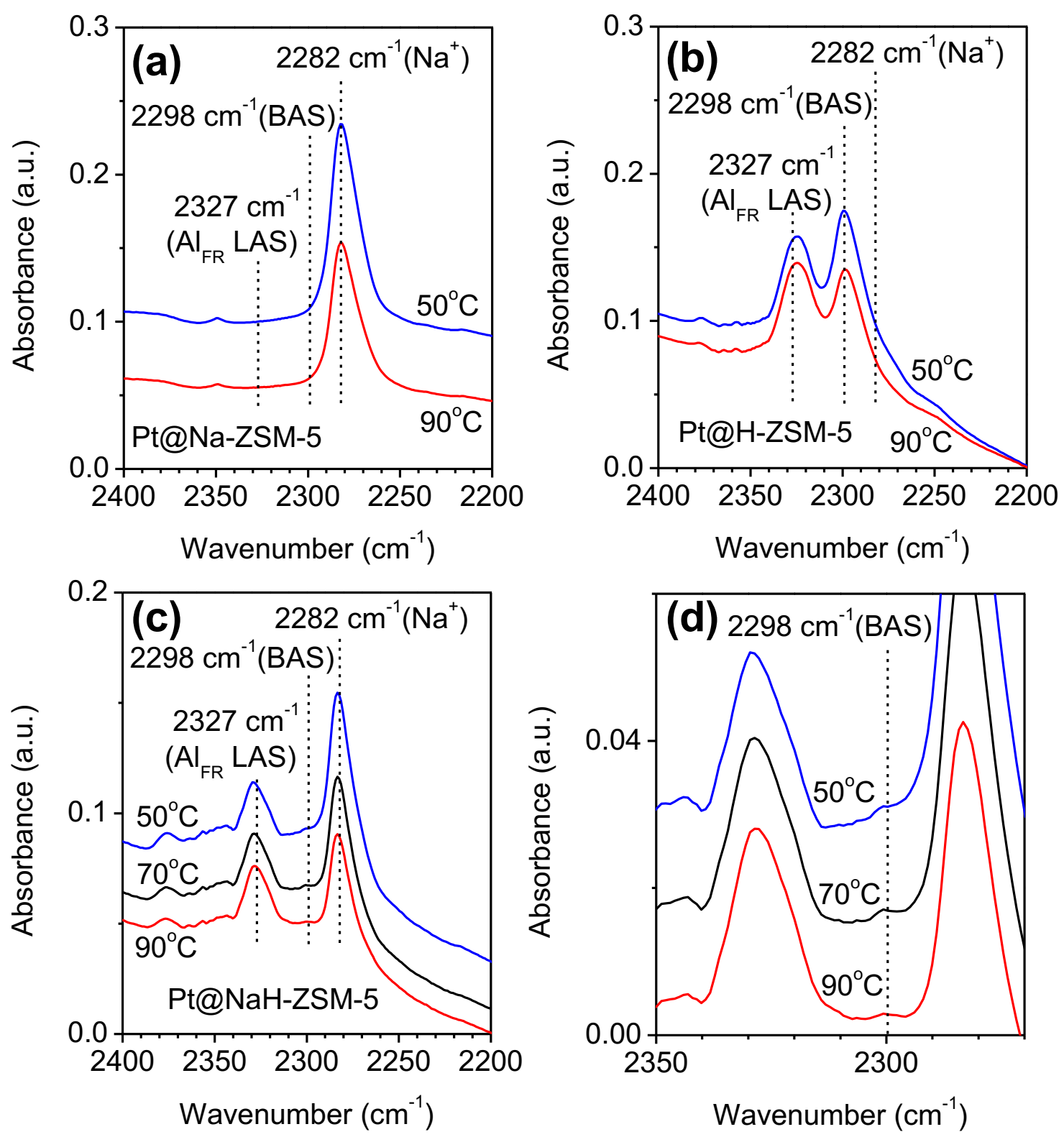

Figure S2. FTIR spectra of (a) Pt@Na-ZSM-5, (b) Pt@H-ZSM-5, (c) Pt@NaH-ZSM-5 samples at $50-90{ }^{\circ} \mathrm{C}$ upon saturation of the samples with $\mathrm{CD}_{3} \mathrm{CN}$ at $30{ }^{\circ} \mathrm{C}$, and (d) zoomed-in spectra of (c). 

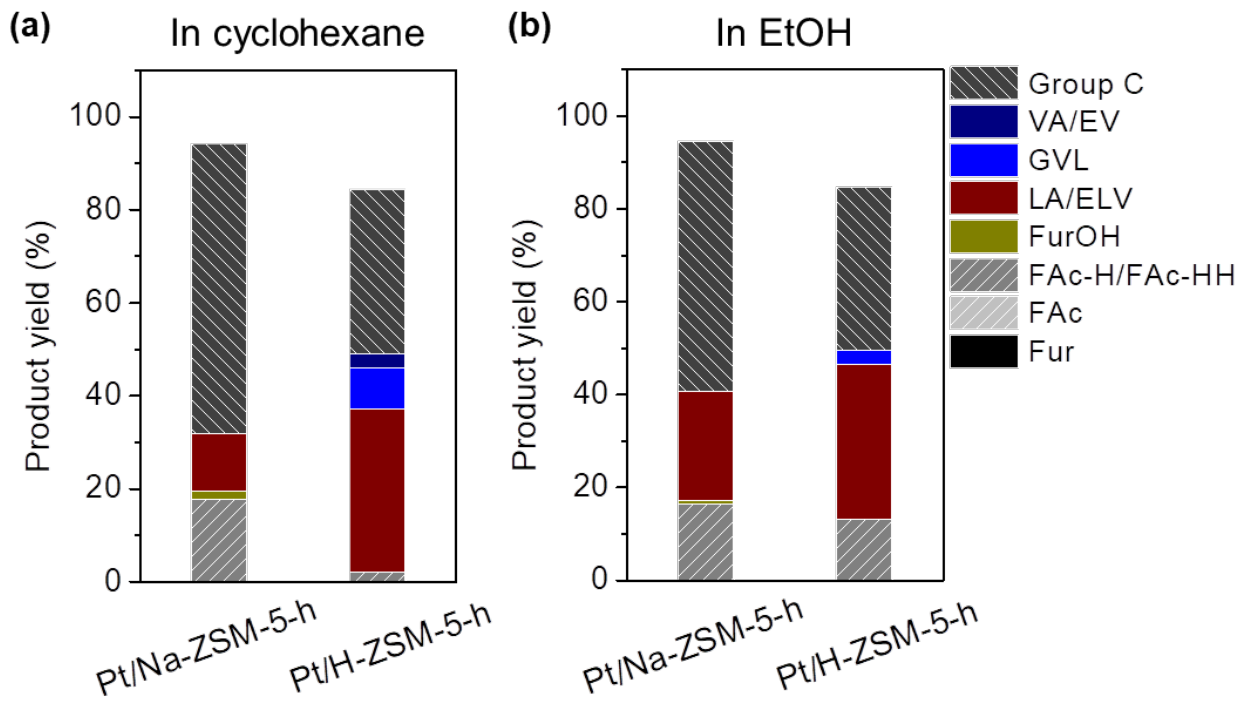

Figure S3. Catalytic performance comparison of Pt/Na-ZSM-5-h and Pt/H-ZSM-5-h for the conversion of furfural in (a) cyclohexane and (b) EtOH solvents. Reaction conditions: $0.5 \mathrm{mmol}$ of furfural, $5 \mathrm{mmol}$ of acetone, $5 \mathrm{mmol}$ of EtOH and $1.5 \mathrm{~mL}$ of cyclohexane $(=13.7 \mathrm{mmol})$ or $\mathrm{EtOH}(=25.4 \mathrm{mmol}) ; 600 \mathrm{psi} \mathrm{H}_{2}$; reaction temperature $160{ }^{\circ} \mathrm{C}$; reaction time $24 \mathrm{~h}$; furfural $/ \mathrm{Al}\left(\mathrm{mol} \mathrm{mol}{ }^{-1}\right)=24$; furfural $/ \mathrm{Pt}\left(\mathrm{mol} \mathrm{mol}^{-1}\right)=616$.

Note: Pt/Na-ZSM-5-h and Pt/H-ZSM-5-h catalysts (Table S1) were prepared by incipient wetness impregnation with an aqueous solution of $\mathrm{H}_{2} \mathrm{PtCl}_{6} \cdot 6 \mathrm{H}_{2} \mathrm{O}$. The impregnated samples with Pt loading of $0.22 \mathrm{wt} \%$ were dried in an oven at $80{ }^{\circ} \mathrm{C}$ overnight, calcined at $500{ }^{\circ} \mathrm{C}$ for $2 \mathrm{~h}$ in air and reduced at $400{ }^{\circ} \mathrm{C}$ for $2 \mathrm{~h}$ under a flow of $\mathrm{H}_{2}\left(10 \mathrm{~mL} \mathrm{~min}^{-1}\right)$ and $\mathrm{N}_{2}\left(90 \mathrm{~mL} \mathrm{~min}^{-1}\right)$. 

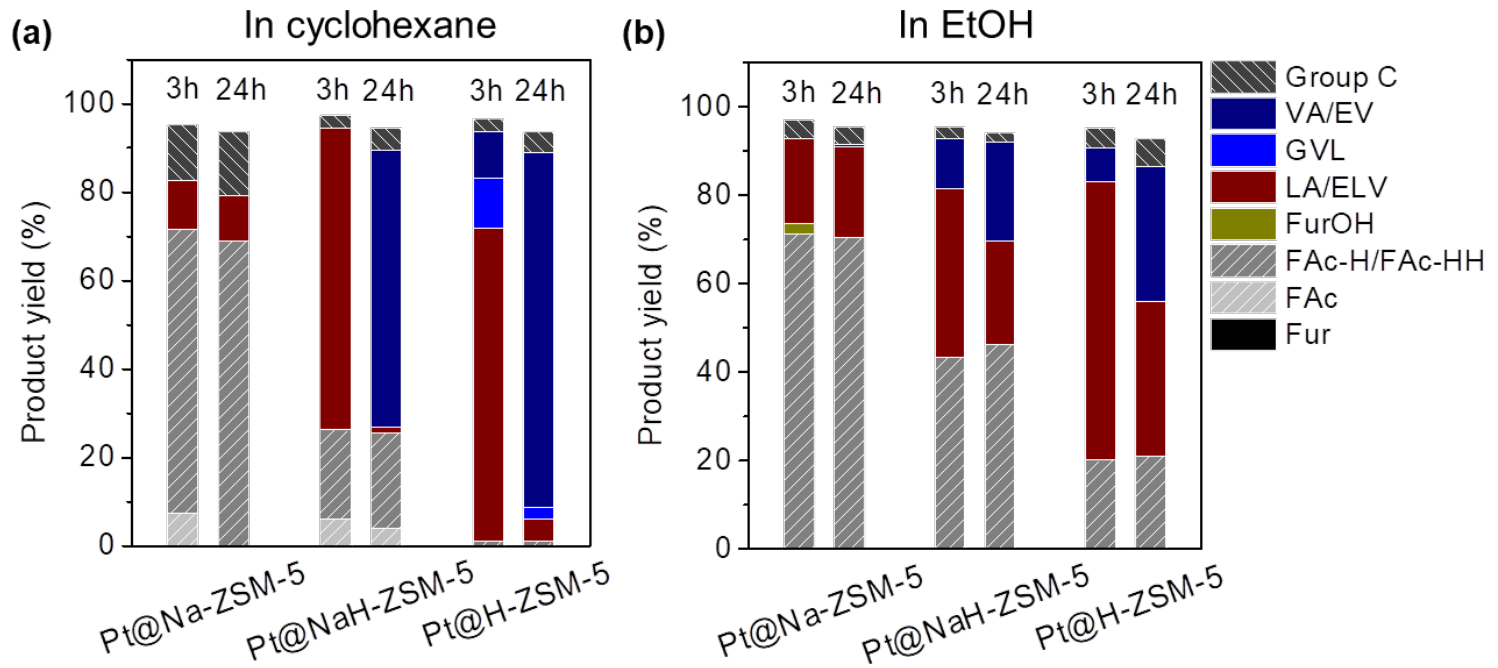

Figure S4. Catalytic performance comparison of Pt@NaH-ZSM-5 with Pt@Na-ZSM-5 and Pt@H-ZSM-5 for the conversion of furfural in (a) cyclohexane and (b) EtOH solvents. Reaction conditions: $0.5 \mathrm{mmol}$ of furfural, $5 \mathrm{mmol}$ of acetone, $5 \mathrm{mmol}$ of EtOH and $1.5 \mathrm{~mL}$ of cyclohexane $(=13.7 \mathrm{mmol})$ or EtOH $(=25.4 \mathrm{mmol}) ; 600 \mathrm{psi}_{2}$; reaction temperature $160{ }^{\circ} \mathrm{C}$; reaction time $3 \mathrm{~h}$ and $24 \mathrm{~h}$; furfural $/ \mathrm{Al}\left(\mathrm{mol} \mathrm{mol}^{-1}\right)=24$; furfural $/ \mathrm{Pt}$ $\left(\mathrm{mol} \mathrm{mol}^{-1}\right)=616$. 
Table S1. Physical and Chemical Properties of Pt@Na-ZSM-5, Pt@NaH-ZSM-5, Pt@H-ZSM-5, Na-ZSM-5-h and H-ZSM-5-h Samples

\begin{tabular}{|c|c|c|c|c|c|c|c|}
\hline Catalyst & $\begin{array}{c}\text { Nominal } \\
\mathrm{Si} / \mathrm{Al} \\
\left(\mathrm{mol} \mathrm{mol}^{-1}\right)\end{array}$ & $\begin{array}{c}\mathrm{Si} / \mathrm{Al}^{a} \\
\left(\mathrm{~mol} \mathrm{~mol}^{-1}\right)\end{array}$ & $\begin{array}{c}\mathrm{Pt} \\
\text { loading }^{a} \\
(\mathrm{wt} \%)\end{array}$ & $\begin{array}{l}\text { Micropore } \\
\text { volume } \\
\left(\mathrm{cm}^{3} \mathrm{~g}^{-1}\right)\end{array}$ & $\begin{array}{c}\mathrm{Pt}^{\mathrm{Pize}}{ }^{c} \\
(\mathrm{~nm})\end{array}$ & $\begin{array}{c}\text { BAS } \\
\text { density }^{d} \\
\left(\mu \mathrm{mol} \mathrm{g}{ }^{-1}\right)\end{array}$ & $\begin{array}{c}\text { LAS } \\
\text { density }^{e} \\
\left(\mu \mathrm{mol} \mathrm{g}{ }^{-1}\right)\end{array}$ \\
\hline Pt@Na-ZSM-5 & 60 & 57 & 0.22 & 0.14 & $5.5(6.3)$ & 75 & 0 \\
\hline Pt@NaH-ZSM-5 & 60 & 57 & 0.22 & 0.14 & (6.4) & 138 & 45 \\
\hline Pt@H-ZSM-5 & 60 & 57 & 0.22 & 0.14 & $5.8(6.4)$ & 204 & 62 \\
\hline Na-ZSM-5-h & 60 & 58 & - & 0.14 & - & 71 & 0 \\
\hline H-ZSM-5-h & 60 & 58 & - & 0.14 & - & 196 & 59 \\
\hline
\end{tabular}

${ }^{a}$ Determined by XRF. ${ }^{b}$ Calculated with the $t$-plot method obtained from $\mathrm{N}_{2}$ physisorption isotherms. ${ }^{c}$ Determined from counting $>100$ particles on TEM images, and determined by CO chemisorption in parentheses under the assumption of a spherical geometry. ${ }^{d}$ Determined by the band at $1545 \mathrm{~cm}^{-1}$ on FTIR spectra with pyridine as the probe at $150{ }^{\circ} \mathrm{C}$. ${ }^{e}$ Determined by the band at $1454 \mathrm{~cm}^{-1}$ on FTIR spectra with pyridine as the probe at $150{ }^{\circ} \mathrm{C}$ and the ratio of integrated molar extinction coefficient of BAS to that of LAS which was reported in earlier literature. ${ }^{1}$

Table S2. Catalytic Results for Size-Selective Oxidation of Cy6-ol and Cy12-ol ${ }^{a}$

\begin{tabular}{lccccc}
\hline Catalyst & $\begin{array}{c}\text { TOF }\left(\mathrm{h}^{-1}\right) \\
\text { for Cy6-ol oxidation }\end{array}$ & $\begin{array}{c}\text { TOF }\left(\mathrm{h}^{-1}\right) \\
\text { for Cy12-ol oxidation }\end{array}$ & $X^{b}$ & $Y^{c}$ & $Z^{d}$ \\
\hline $\mathrm{Pt} @ \mathrm{Na}-Z \mathrm{ZSM}-5$ & 32.6 & 1.1 & 29.6 & 11.9 & 0.92 \\
$\mathrm{Pt} @ \mathrm{H}-\mathrm{ZSM}-5$ & 32.4 & 1.1 & 29.5 & 11.8 & 0.92 \\
$\mathrm{Pt} / \mathrm{SiO}_{2}$ & 36.2 & 14.5 & 2.50 & - & - \\
\hline
\end{tabular}

${ }^{a}$ Reaction conditions are as follows: $0.1 \mathrm{mmol}$ of Cy6-ol or Cy12-ol; $0.3 \mathrm{mmol}$ TBHP; $2 \mathrm{~mL}$ acetonitrile; Cy6-ol/Pt $\left(\mathrm{mol} \mathrm{mol}^{-1}\right)=222 ; \mathrm{Cy} 12-\mathrm{ol} / \mathrm{Pt}\left(\mathrm{mol} \mathrm{mol}^{-1}\right)=111$; reaction temperature $60{ }^{\circ} \mathrm{C}$.

${ }^{b} X_{\text {sample }}=\mathrm{TOF}_{\text {Cy6-ol }} / \mathrm{TOF}_{\text {Cy12-ol }}$.

${ }^{c} Y_{\text {sample }}=X_{\text {sample }} / X_{\mathrm{Pt} / \mathrm{SiO} 2}$.

${ }^{d} Z_{\text {sample }}=1-1 / Y_{\text {sample. }}$ 
Table S3. Catalyst Performances for Tandem Reactions Associated with Furfural Conversion in Earlier Literature

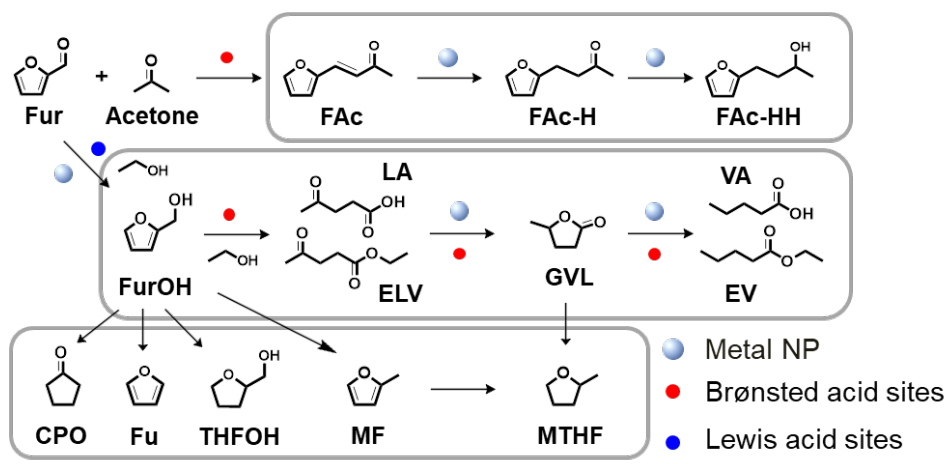

\begin{tabular}{|c|c|c|c|c|}
\hline Tandem reaction & Catalyst & $\begin{array}{l}\text { Reaction } \\
\text { conditions }\end{array}$ & Target products $^{a}$ & Ref. \\
\hline Fur to VA/EV & Pt@H-ZSM-5 & $160^{\circ} \mathrm{C}, 24 \mathrm{~h}$ & VA/EV $(80 \%)$ & This work \\
\hline Fur to FAc-H/FAc-HH & Pt@Na-ZSM-5 & $160^{\circ} \mathrm{C}, 24 \mathrm{~h}$ & FAc-H/FAc-HH (69\%) & This work \\
\hline Fur to VA/EV & Pt@H-ZSM-5 & $140^{\circ} \mathrm{C}, 24 \mathrm{~h}$ & VA/EV (86\%) & Cho et al. ${ }^{2}$ \\
\hline Fur to FAc-H/FAc-HH & Pt@Na-ZSM-5 & $180^{\circ} \mathrm{C}, 3 \mathrm{~h}$ & FAc-H/FAc-HH (87\%) & Cho et al. ${ }^{3}$ \\
\hline Fur to $\mathrm{Fu}$ & Pd@S-1-OH-10 & $250-275^{\circ} \mathrm{C}$ & $\mathrm{Fu}(99 \%)$ & Wang et al. ${ }^{4}$ \\
\hline Fur to MF & $\alpha-\mathrm{MoC}$ & $150^{\circ} \mathrm{C}, 6 \mathrm{~h}$ & MF $(90 \%)$ & Deng et $\mathrm{al}^{5}$ \\
\hline Fur to FurOH & Pt-CeO ${ }_{2} @$ UIO-66-NH ${ }_{2}$ & $80^{\circ} \mathrm{C}, 30 \mathrm{~h}$ & FurOH (99\%) & Long et al. ${ }^{6}$ \\
\hline Fur to FurOH & Cu-MOF & $130^{\circ} \mathrm{C}, 3 \mathrm{~h}$ & FurOH (97\%) & Chen et al. ${ }^{7}$ \\
\hline Fur to THFOH & $\mathrm{Ni} / \mathrm{MMO}-\mathrm{CO}_{3}$ & $110^{\circ} \mathrm{C}, 3 \mathrm{~h}$ & THFOH (99\%) & Meng et al. ${ }^{8}$ \\
\hline Fur to GVL & $\mathrm{Au} / \mathrm{ZrO}_{2}+\mathrm{ZSM}-5$ & $120^{\circ} \mathrm{C}, 30 \mathrm{~h}$ & GVL $(80 \%)$ & Zhu et al. ${ }^{9}$ \\
\hline
\end{tabular}

${ }^{a}$ Yields of target products in parenthesis. 
Table S4. Conversion Rate of Furfural and Formation Rates of Group A, Group B and Group C Products in the Conversion of Furfural on Pt@Na-ZSM-5, Pt@HZSM-5 and H-ZSM-5-h in Cyclohexane Solvent ${ }^{a}$

\begin{tabular}{|c|c|c|c|c|c|c|}
\hline Entry & Catalyst & $\begin{array}{l}\text { Type } \\
\text { of gas }\end{array}$ & $\begin{array}{c}\text { Fur } \\
\text { conversion rate } \\
\left(\mathrm{mmol}^{b}\right. \\
\left.\text { g-cat }^{-1} \mathrm{~h}^{-1}\right)\end{array}$ & $\begin{array}{c}\text { Group A } \\
\text { formation rate } \\
(\mathrm{mmol} \\
\left.\text { g-cat }^{-1} \mathrm{~h}^{-1}\right)\end{array}$ & $\begin{array}{c}\text { Group B } \\
\text { formation rate } \\
(\mathrm{mmol} \\
\left.\text { g-cat }{ }^{-1} \mathrm{~h}^{-1}\right)\end{array}$ & $\begin{array}{c}\text { Group C } \\
\text { formation rate } \\
\left(\mathrm{mmol}^{b}\right. \\
\left.\text { g-cat }^{-1} \mathrm{~h}^{-1}\right)\end{array}$ \\
\hline 1 & Pt@Na-ZSM-5 & $\mathrm{H}_{2}$ & 23.0 & 18.5 & 1.5 & 0.0 \\
\hline 2 & Pt@Na-ZSM-5 & $\mathrm{N}_{2}$ & 7.8 & 5.3 & 0.0 & 0.0 \\
\hline 3 & Pt@H-ZSM-5 & $\mathrm{H}_{2}$ & 42.0 & 0.5 & 38.0 & 0.0 \\
\hline 4 & Pt@H-ZSM-5 & $\mathrm{N}_{2}$ & 16.3 & 3.8 & 11.7 & 0.0 \\
\hline 5 & H-ZSM-5-h & $\mathrm{N}_{2}$ & 16.0 & 3.7 & 11.2 & 0.0 \\
\hline
\end{tabular}

${ }^{a}$ Reaction conditions: $0.5 \mathrm{mmol}$ of furfural, $5 \mathrm{mmol}$ of acetone, $5 \mathrm{mmol}$ of EtOH and $1.5 \mathrm{~mL}$ of cyclohexane; 600 psi $\mathrm{H}_{2}$ of $\mathrm{N}_{2}$; reaction temperature $160{ }^{\circ} \mathrm{C}$; reaction time $30 \mathrm{~min}$. ${ }^{a}$ Rates are defined as mmol of furfural consumption or product formation divided by catalyst weight $(\mathrm{g})$ per reaction time $(\mathrm{h})$, which are determined when furfural conversion lies below $10 \%$. 
Table S5. Product Distribution in the Conversion of Furfural on Pt@Na-ZSM-5, Pt@H-ZSM-5 and Pt@NaH-ZSM-5 Catalysts in Different Solvents ${ }^{a}$

\begin{tabular}{ccccccc}
\hline Entry & Catalyst & Solvent & $\begin{array}{c}\text { Reaction } \\
\text { time (h) }\end{array}$ & $\begin{array}{c}\text { Group A } \\
\text { yields (\%) }\end{array}$ & $\begin{array}{c}\text { Group B } \\
\text { yields (\%) }\end{array}$ & $\begin{array}{c}\text { Group C } \\
\text { yields (\%) }\end{array}$ \\
\hline 1 & Pt@Na-ZSM-5 & Cyclohexane & 3 & 71.7 & 11.0 & 12.5 \\
2 & & Cyclohexane & 24 & 69.0 & 10.1 & 14.5 \\
3 & & EtOH & 3 & 71.2 & 21.6 & 4.1 \\
4 & & EtOH & 24 & 70.5 & 21.0 & 4.0 \\
5 & Pt@H-ZSM-5 & Cyclohexane & 3 & 1.0 & 92.8 & 2.9 \\
6 & & Cyclohexane & 24 & 1.0 & 87.9 & 4.8 \\
7 & & EtOH & 3 & 20.1 & 70.7 & 4.3 \\
8 & & EtOH & 24 & 21.1 & 65.4 & 6.3 \\
9 & Pt@NaH-ZSM-5 & Cyclohexane & 3 & 26.3 & 68.2 & 0.0 \\
10 & & Cyclohexane & 24 & 25.5 & 68.0 & 0.0 \\
11 & & EtOH & 3 & 43.5 & 49.4 & 2.4 \\
12 & & EtOH & 24 & 44.0 & 48.1 & 2.0 \\
\hline
\end{tabular}

${ }^{a}$ Reaction conditions: $0.5 \mathrm{mmol}$ of furfural, $5 \mathrm{mmol}$ of acetone, $5 \mathrm{mmol}$ of $\mathrm{EtOH}$ and $1.5 \mathrm{~mL}$ of cyclohexane $(=13.7 \mathrm{mmol})$ or $\mathrm{EtOH}(=25.4 \mathrm{mmol}) ; 600 \mathrm{psi} \mathrm{H}_{2}$; reaction temperature $160{ }^{\circ} \mathrm{C}$; reaction time $3 \mathrm{~h}$ and $24 \mathrm{~h}$; furfural $/ \mathrm{Al}\left(\mathrm{mol} \mathrm{mol}^{-1}\right)=24$; furfural $/ \mathrm{Pt}\left(\mathrm{mol} \mathrm{mol}^{-1}\right)=616$. 
Table S6. Estimation of Water Amount Produced from EtOH Etherification during the Conversion of Furfural ${ }^{a}$

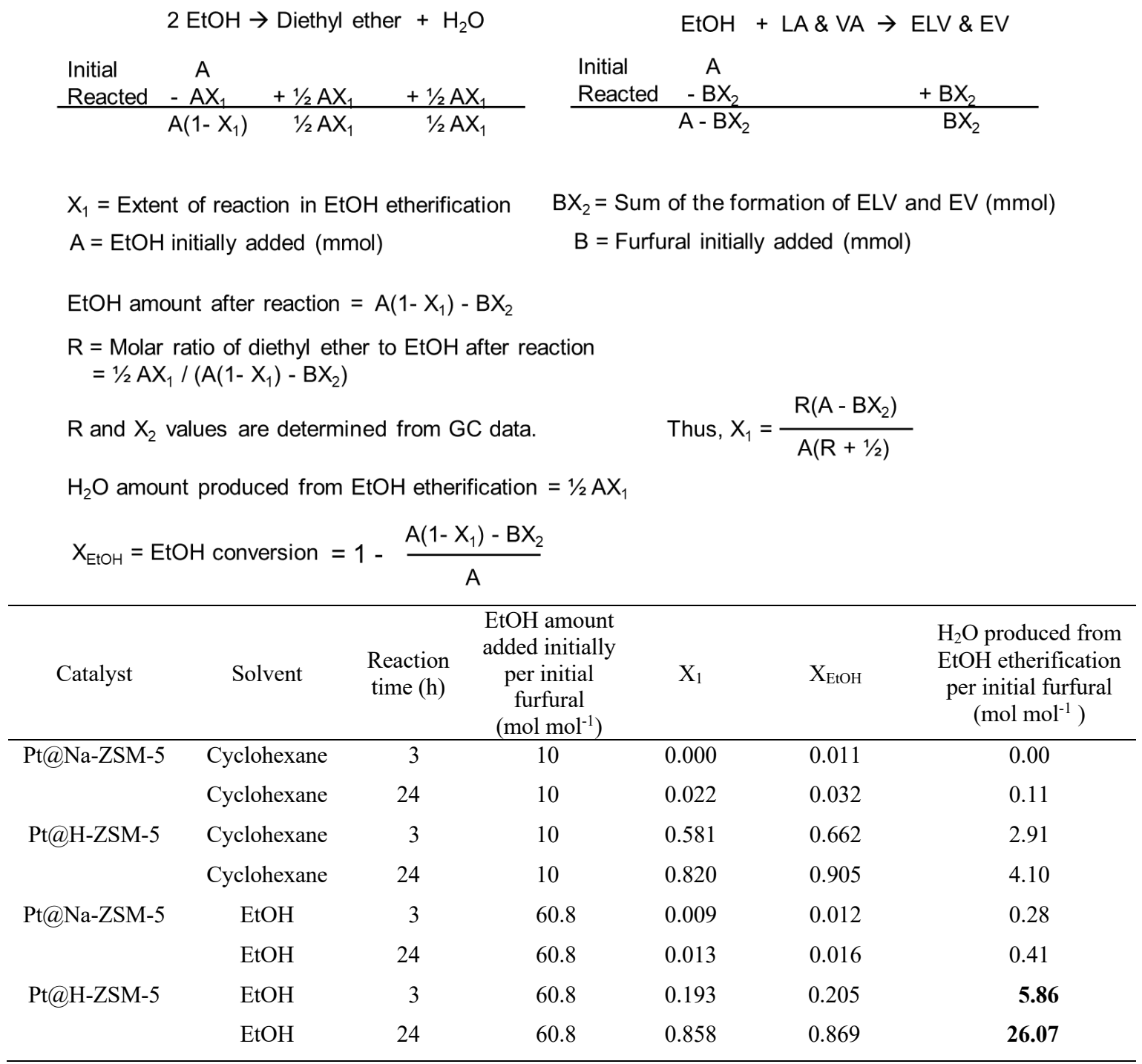

${ }^{a}$ Reaction conditions: $0.5 \mathrm{mmol}$ of furfural, $5 \mathrm{mmol}$ of acetone, $5 \mathrm{mmol}$ of $\mathrm{EtOH}$ and $1.5 \mathrm{~mL}$ of cyclohexane $(=13.7 \mathrm{mmol})$ or EtOH $(=25.4 \mathrm{mmol}) ; 600 \mathrm{psi}_{2}$; reaction temperature $160{ }^{\circ} \mathrm{C}$; reaction time $3 \mathrm{~h}$ and $24 \mathrm{~h}$; furfural $/ \mathrm{Al}\left(\mathrm{mol} \mathrm{mol}^{-1}\right)=24$; furfural $/ \mathrm{Pt}\left(\mathrm{mol} \mathrm{mol}^{-1}\right)=616$. 
Table S7. Effect of Water or EtOH Initially Added on the Conversion of Furfural with Pt@H-ZSM-5 in Cyclohexane Solvent ${ }^{a}$

\begin{tabular}{ccccccc}
\hline Catalyst & Solvent & $\begin{array}{c}\mathrm{H}_{2} \mathrm{O} \text { amount } \\
\text { added } \\
\text { initially per } \\
\text { initial furfural } \\
\left(\mathrm{mol} \mathrm{mol}^{-1}\right)\end{array}$ & $\begin{array}{c}\mathrm{EtOH} \text { amount } \\
\text { added } \\
\text { initially per } \\
\text { initial furfural } \\
\left(\mathrm{mol} \mathrm{mol}^{-1}\right)\end{array}$ & $\mathrm{X}_{1}{ }^{b}$ & $\mathrm{X}_{\mathrm{EtOH}}{ }^{c}$ & $\begin{array}{c}\mathrm{H}_{2} \mathrm{O} \text { produced } \\
\text { from EtOH } \\
\text { etherification } \\
\text { per initial furfural } \\
\left(\text { mol mol }^{-1}\right)\end{array}$ \\
\hline Pt@H-ZSM-5 & Cyclohexane & 0 & 10 & 0.820 & 0.905 & 4.10 \\
& Cyclohexane & 5 & 10 & 0.810 & 0.896 & 4.05 \\
& Cyclohexane & 15 & 10 & 0.790 & 0.879 & 3.95 \\
& Cyclohexane & 25 & 10 & 0.720 & 0.801 & 3.60 \\
& Cyclohexane & 30 & 10 & 0.709 & 0.790 & 3.54 \\
& Cyclohexane & 0 & 0 & 0.000 & 0.000 & 0.00 \\
& Cyclohexane & 0 & 1 & 0.303 & 0.554 & 0.15 \\
& Cyclohexane & 0 & 5 & 0.457 & 0.587 & 1.14 \\
Cyclohexane & 0 & 10 & 0.820 & 0.905 & 4.10 \\
Cyclohexane & 0 & 15 & 0.822 & 0.878 & 6.17 \\
Cyclohexane & 0 & 25 & 0.844 & 0.881 & 10.60 \\
Cyclohexane & 0 & 35 & 0.838 & 0.863 & 14.67 \\
Cyclohexane & 0 & 40 & 0.855 & 0.878 & 17.10 \\
\hline
\end{tabular}

${ }^{a}$ Reaction conditions: $0.5 \mathrm{mmol}$ of furfural, $5 \mathrm{mmol}$ of acetone, $1.5 \mathrm{~mL}$ of cyclohexane $(=13.7$ mmol), and either $0-15 \mathrm{mmol}$ of $\mathrm{H}_{2} \mathrm{O}$ with $5 \mathrm{mmol}$ of EtOH or 0-20 mmol of EtOH; $600 \mathrm{psi}_{2}$; reaction temperature $160{ }^{\circ} \mathrm{C}$; reaction time $24 \mathrm{~h}$; furfural $/ \mathrm{Al}\left(\mathrm{mol} \mathrm{mol}^{-1}\right)=24$; furfural $/ \mathrm{Pt}(\mathrm{mol}$ $\left.\mathrm{mol}^{-1}\right)=616 .{ }^{b}$ Defined as extent of reaction in the EtOH etherification, as described in Table S4. ${ }^{c}$ Defined as EtOH conversion, as described in Table S4. 


\section{References}

(1) Emeis, C. A. Determination of Integrated Molar Extinction Coefficients for InfraredAbsorption Bands of Pyridine Adsorbed on Solid Acid Catalysts. J. Catal. 1993, 141, 347-354.

(2) Cho, H. J.; Kim, D. Y.; Li, S.; Su, D.; Ma, D.; Xu, B. Molecular Level Proximity of Metal and Acid Sites in Zeolite Encapsulated Pt Nanoparticles for Selective Multistep Tandem Catalysis. ACS Catal. 2019, 10, 3340-3348.

(3) Cho, H. J.; Kim, D.; Li, J.; Su, D.; Xu, B. J. Zeolite-Encapsulated Pt Nanoparticles for Tandem Catalysis. J. Am. Chem. Soc. 2018, 140, 13514-13520.

(4) Wang, C.; Liu, Z.; Wang, L.; Dong, X.; Zhang, J.; Wang, G.; Han, S.; Meng, X.; A., Z.; Xiao, F. S. Importance of Zeolite Wettability for Selective Hydrogenation of Furfural over Pd@Zeolite Catalysts. ACS Catal. 2018, 8, 474-481.

(5) Deng, Y.; Gao, R.; Lin, L.; Liu, T.; Wen, X. D.; Wang, S.; Ma, D. Solvent Tunes the Selectivity of Hydrogenation Reaction over $\alpha$-MoC Catalyst. J. Am. Chem. Soc. 2018, 140, 14481-14489.

(6) Long, Y.; Song, S.; Li, J.; Wu, L.; Wang, Q.; Liu, Y.; Jin, R.; Zhang, H. Pt/CeO2@MOF Core@Shell Nanoreactor for Selective Hydrogenation of Furfural via the Channel Screening Effect. ACS Catal. 2018, 8, 8506-8512.

(7) Chen, K.; Ling, J. L.; Wu, C. D. In Situ Generation and Stabilization of Accessible $\mathrm{Cu} / \mathrm{Cu} 2 \mathrm{O}$ Heterojunctions inside Organic Frameworks for Highly Efficient Catalysis. Angew. Chem. Int. Ed. 2020, 132, 1941-1947.

(8) Meng, X.; Yang, Y.; Chen, L.; Xu, M.; Zhang, X.; Wei, M. A Control over Hydrogenation Selectivity of Furfural via Tuning Exposed Facet of Ni Catalysts. ACS Catal. 2019, 9, 4226-4235.

(9) Zhu, S. H.; Xue, Y. F.; Guo, J.; Cen, Y. L.; Wang, J. G.; Fan, W. B. Integrated Conversion of Hemicellulose and Furfural into gamma-Valerolactone over $\mathrm{Au} / \mathrm{ZrO} 2$ Catalyst Combined with ZSM-5. ACS Catal. 2016, 6, 2035-2042. 